\title{
Monitoração contínua da glicose intersticial em cães sadios
}

Karina Casagrande FERREIRA ${ }^{1}$ Márcia Marques JERICÓ ${ }^{2}$; Achille Marcelo de PIETRO' Elídia Zotelli dos SANTOS';

Liliam SEWASTJANOW'; Talita LEITE'; Juliana BAPTISTA
Rua Antonio Estigarribia, 130 EP 02556-030. Sao Paulo/SP, Brasil

\ karina.casagande@outlook.com

\section{Continuous monitoring of interstitial glucose in healthy dogs}

\section{Resumo}

A manutenção da glicose corpórea em niveis adequados é primordi para o controle da homeostase, oferecendo substrato energético fundamental para o bom funcionamento organicico. Para a mensuraçẵo $d$ gilcose corporrea, estão disponiveis técnicas de avaliaçōes portateieis e laboratoriais. Assim, visando a futuros estudos clinicos das variaçōes da glicose corporea em cáes, objetivou-se observar a exequibilidade de um sistema de monitoramento continuo da glicose intersticial denominado CGMS® (Continuous Glucose Monitoring System, Sistema Guardia onic /E.U.A.). O grupo de estudo foi conpc oito cães clinicamente hígidos, e todos os animais estuddados apresentaram os maiores valores de glicose intersticial durante o processo $\mathrm{d}$ contenção e obtenção da amostra de sangue venoso para a calibração do aparelho, e os menores valores durante o periodo noturno devido glicose venosa e intersticial, realizadas em um mesmo momento, apresentou uma boa concordancla entresi. Os valoes de medlana de gilcose obtidos via venosa foram $82,5 \mathrm{mg} / \mathrm{dL}, 81 \mathrm{mg} / \mathrm{dL} ; 80 \mathrm{mg} / \mathrm{dL} ; 78 \mathrm{mg} / \mathrm{d}$; $86,5 \mathrm{mg} / \mathrm{dL}, 83 \mathrm{mg} / \mathrm{dL}, 84 \mathrm{mg} / \mathrm{dL} ; 99,5 \mathrm{mg} / \mathrm{dL}$. Já as que foram avaliad pel..CGMS®, corresponderam a 77mg/dL; $77 \mathrm{mg} / \mathrm{dL} ; 69 \mathrm{mg} / \mathrm{dl} ; 80 \mathrm{mg} /$ dL; 81,5mg/dL; $76 \mathrm{mg} / \mathrm{dL} ; 52,5 \mathrm{mg} / \mathrm{dL} ;$ e 86mg/dL. Houve situaçōes pontuais de não registro de dados por remoção do sensor e de reação cutânea inflamatónia leve no local de fixação. Os resultados obtidos confirmaram a eficácia do CGMS $\circledast$ na avaliação da gicose intersticial em âmbito doméstico e assistido, evidenciando, também, a necessidade de treinamento do proprietário quanto ao manuseio do aparelho. ausência de manipulação. Também foi observado que a comparação da

\section{Summary}

The maintenance of body glucose in proper levels is of paramount

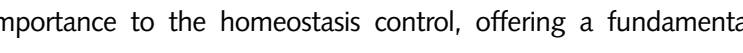
pergelic substrate for the proper organic performance. For the purposes of measuing body glucose levels, there are avaliable portable and laboratory assessment techniques. Therefore, aiming at future cinical trials on body glucose variation in dogs, it had the purpose of observing the feasibility of a continuous interstitial glucose monitoring system named CGMS® (Continuous Glucose Monitoring System, Guardian Real-Time System/Medronic/U.S.A.) Eight clinically healthy dogs composed the study group and all of them presented the high intersitital glucose values during the contention and obtaining process

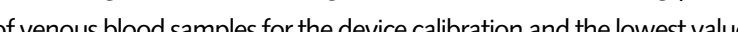
performed the conprison of venous and interst ty at the same nomet and presting a god ag the same moment and presenting a good agreement between The

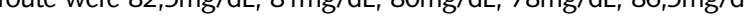
corresponded.to $77 \mathrm{mg} / \mathrm{dL} ; 77 \mathrm{mg} / \mathrm{dL} ; 69 \mathrm{mg} / \mathrm{dL} ; 80 \mathrm{mg} / \mathrm{dL} ; 81,5 \mathrm{mg} / \mathrm{dL}$ $76 \mathrm{mg} / \mathrm{dL} ; 52,5 \mathrm{mg} / \mathrm{dL} ; \mathrm{e} 86 \mathrm{mg} / \mathrm{dL}$. There has been certain situations of non-recording of data by removal of the sensor and light inflammatory cutaneous reaction on the fixation site. Results obtained confirmed the efficacy of CGMS $\Theta$ on the assessment of interstitial glucose with the home settings and assisted, moreover also evidencing the need of training to the device owner regarding its related handling.

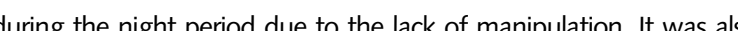
$83 \mathrm{mg} / \mathrm{dL}, 84 \mathrm{mg} / \mathrm{dL}, 99,5 \mathrm{mg} / \mathrm{dL}$ whereas those.evaluated.by.CCMSO

8

Palauras-chave
Clicemia. Clicosimetro. Venosa. Sensor. Calibraçăa.

Keywords

Blood glucose. Glucometer.
Venous. Sensor. Calibration.

Recebido em 15 de novembro de 2014 e aprovado em 07 de agosto de 2015. Premiado em primeir lugar no Congresso Paulista das Especialidades 2014
1. Discente de graduação de Medicina Veterinária da Universidade Anhembi Morumbi. 2. Professora orrentadora Doutora da Clinica de Pequenos Animais da Universidade Anhembi Morumb glicose é um carboidrato monossacarídeo, que representa a principal fonte de energia para os animais, além disso, é a única fonte de energia para as hemácias. A glicose é carregada para o interior licose (GLUT), em seguida, essa substância passa por inúmeras transformações até a geração da energia necessária para o metabolismo aeróbico (BERG; TYMOCZKO; STRYER, 2007). A transformação de glicose em energia exige a perfeita interacão entre alguns mediadores químicos, principalmente os hormônios pancreáticos, insulina e glucagon (NELSON; SALISBURY, 1998).

A avaliação do estado glicêmico do paciente pode ser realizada por diferentes metodologias. Geralmente, a glicose corpórea dos animais é mensurada pela glicose sanguínea venosa, ou glicemia, por meio de glicosímetros portáteis ou testes laboratoriais de bancada (análise bioquímica). Considera-se que o cão é hiperglicêmico quando a sua concentração de glicose no sangue é maior que $130 \mathrm{mg} / \mathrm{dL}$ e hipoglicêmico quando menor que $60 \mathrm{mg} / \mathrm{dL}$ (SERÔDIO; CARVALHO; MACHADO, 2008). 
O controle da glicose venosa de forma seriada, investigação e manejo de doenças, representando um instambém conhecido por curva glicêmica é definido atualmente como técnica de padrão ouro para o controle e tratamento de animais com variações importantes na glicemia, muito embora os valores de glicemia obtidos possam ser influenciados por fatores externos, em especial os causados pela manipulação do animal. Para se
obter uma curva glicêmica, torna-se necessária a obtenção de sangue por meio de punção venosa repetida, ou
cor por cateteres fixados, a cada duas a três horas, por um por cateteres fixados, a cada duas a tres horas, por um período minimo de 12 horas. Esta forma invasiva de obtenção de dados geralmente resulta em alteração da rotina do paciente e também pode levar à hiperglicemia induzida por estresse. Do exposto depreende-se que, as curvas glicêmicas nem sempre podem corresponder às oscilações reais de glicemia no dia a dia. (WIEDMEYER; DECLUE, 2011). A mensuração da concentração de glicose a partir do fluido intersticial pode ser vantajosa, pois minimiza a necessidade de venopuntura e além disso a concentração de glicose no fluido intersticial é comparável com a mediç̃ vel com a medição da glicose no sangue total ou plas

Esse tipo de sistema de monitoraço conin) Esse tipo de sistema de monitoraça continua de glicose permite o acompanhamento panorâmico das flutuações diárias, determinando as concentrações de glicose intersticial por meio de uma técnica pouco invasiva que consiste na inserção de um pequeno sensor flexível no tecido subcutâneo do animal, que fica conectado a um aparelho de registro eletrônico das mensurações. O sistema de monitoramento desenvolvido no presente trabalho utiliza o método de análise por sensor enzimático, que corresponde a um fio de metal recoberto por uma camada enzimática que ao reagir com a glicose intersticial acarreta a oxidaç̃ o da glicose presente no interstício cutâneo result ema untersticio

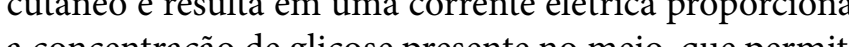
que o leitor de glicose intersticial registre os valores seriaque o leitor de glicose intersticial regist
dos de glicose (MURAKAMI, 2007).

Em metodologia já validada para cães, gatos e cavalos há mais de uma década, as medidas da glicose intersticial há mais de uma década, as medidas da glicose intersticial
são realizadas a cada cinco minutos resultando em 288 são realizadas a cada cinco minutos resultando em 288 avaliados em um programa de computador que gerara um gráfico de distribuição dos valores em um espaço de tempo. Este sistema evita situações de estresse que podem prejudicar a avaliação adequada do perfil glicêmico, como internação em ambiente hospitalar sedação e flebotomias seriadas (WIEDMEYER et al., 2005).

Na medicina humana, a maioria dos autores concorda que a mensuração constante de glicose intersticial é um procedimento pouco invasivo e de grande valia na trumento importante para o diagnóstico e tratamento d

Em cães, os principais benefícios da avaliação contínua e glicose intersticial, são a possibilidade da identificação de momentos episódicos de oscilações glicêmicas e a posibilidade de serem obtidos valor período noturno (SURMAN; FLEEMAN, 2013).

No Brasil, ainda não foram realizadas pesquisas mpregando o CGMS para a avaliação da glicemia en animais sadios ou doentes. Assim, o presente trabalh padronizou o método para a realidade nacional, a part de sua utilização em cães hígidos, mantidos em ambiente controlado (canil) e em ambiente doméstico, de forma vando futuras aplicações para a monitoração clínica das variações da glicose corporea em cães.

\section{Material e métodos}

Foram utilizados oito animais clinicamente hígidos quatro fêmeas e quatro machos, com idade média de quatro anos, dos quais cinco sem raça definida e três com definição racial, dois não eram gonadectomizados. Os animais foram selecionados após a realização de um exame clinico completo (incluindo exames laboratoria de rotina), e escolhidos de forma a representar dois tipo de ambiente: os mantidos em ambiente controlado (cães do canil do Hospital Veterinário Escola) e os mantidos em ambiente doméstico, com a aprovação consensual dos tutores e cuidadores.

Os fatores de exclusão foram a presença de doenças sistemicas ou a ocorrência de terapia medicamentosa crônica ou recente.

A partir da seleção, os cães foram submetidos ao uso do sistema CGMS', (Continuous Glucose Monitoring System, Sistema Guardian Real-Time'/Medtronic (E.U.A.), equipamento que dispõe de um sensor ampe-

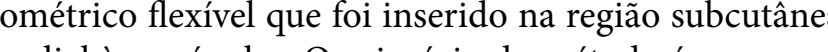
medial às escápulas. $O$ princípio do método é mensura glicose com base na reação eletroquímica da enzim valores, entre 40 e $400 \mathrm{mg} / \mathrm{dl}$, eram captados a cad 10 segundos, com o registro da média desses valores a cada cinco minutos,num total de 288 mensurações em 24 horas e 864 medidas durante 72 horas. Esses resultados foram transmitidos, por um sistema de transmissão sem fio (Minilink Wireless') a um monitor que era resa gicose, ben como, pela tran pacientes (ABDALLA, 2007). simular condições ideais e de rotina domiciliar, objetiglicose-oxidase com a glicose do fluido intersticial. Os ponsável pelo armazenamento das leituras coe era res-
Para calibração e funcionamento do aparelho, é necessário o registro, ou inserção, no mesmo, de um valor de glicemia obtida por venopunção, a cada 12 horas. Sendo que no primeiro dia, essa regulação era iniciada duas horas após a inicialização do aparelho e, novamente, seis horas após a prim cara calibraço O aparelho utilize para a medição da glicemia foi o glicosímetro Bayer Modelo Breeze ${ }^{\mathrm{m}} 2$.

Os resultados obtidos foram expressos em mediana, mínimo e máximo. Para análise estatística, foi utilizado o teste $\mathrm{T}$ de Student para comparação entre os valores de glicose venosa e intersticial, respectivamente, obtidos pelo glicosímetro e GCMS". Também foi utilizado o método de Wilcoxon para a comparação entre os valores pelo GCMS obtidos em um mesmo animal, e Mann-Whitney para a comparação entre os valores do GCMS ${ }^{\circ}$ de diferentes animais. Foram considerados significantes os resultados cujo valores de $p$ fossem menores que $0,05(p<0,05)$.

Foram desenvolvidos vestimentas e aparatos específicos para uma melhor fixaç̃o e uso do apare pomopara ofecer conforto uos donimistestados (Figua 1

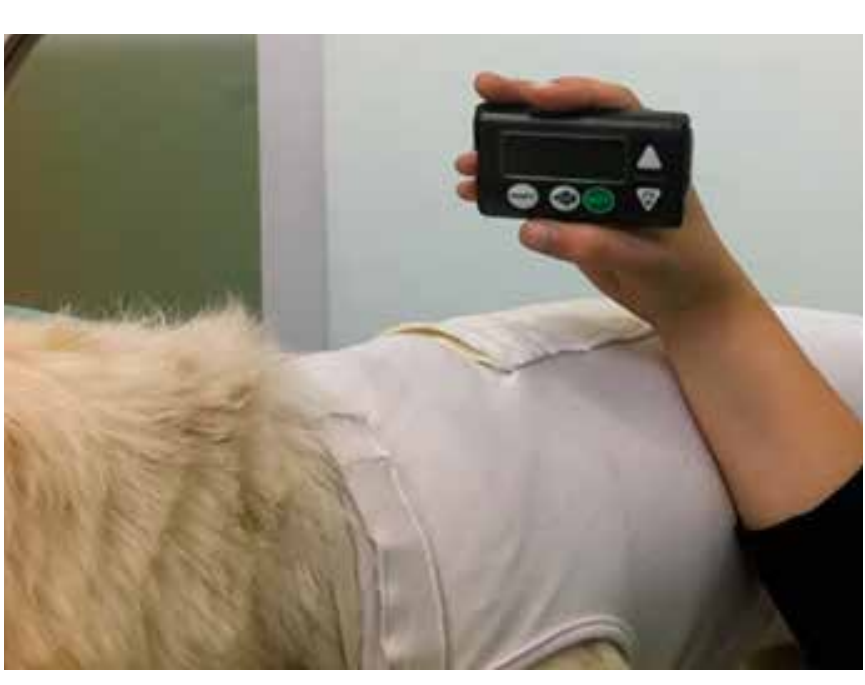
ao animale proteçăo do sensor
Fonte: Casagrande, K. (2014)

Este projeto científico foi submetido, aprovado e upervisionado pelo Comitê de Ética da Universidade Anhembi Morumbi, os animais foram acompanhados diuturnamente pelos responsáveis do projeto, funcionários, e pelos respectivos tutores quando em acompanhamento domiciliar.

Resultados/Discussão

Referente às 72 horas de monitoração, os menores valores individuais de glicose intersticial registrados nos oito animais foram de $48 \mathrm{mg} / \mathrm{dL}, 40 \mathrm{mg} / \mathrm{dL}, 40 \mathrm{mg} / \mathrm{dL}$ $40 \mathrm{mg} / \mathrm{dL}, 48 \mathrm{mg} / \mathrm{dL}, 40 \mathrm{mg} / \mathrm{dL}, 40 \mathrm{mg} / \mathrm{dL}$ e $40 \mathrm{mg} / \mathrm{dL}$ respectivamente, sendo que a mediana foi de $40 \mathrm{mg} /$ d. Os maiores resultados registrados foram $102 \mathrm{mg} / \mathrm{dL}$ $108 \mathrm{mg} / \mathrm{dL}, 94 \mathrm{mg} / \mathrm{dL}, 108 \mathrm{mg} / \mathrm{dL}, 112 \mathrm{mg} / \mathrm{dL}, 102 \mathrm{mg} / \mathrm{dL}$ $114 \mathrm{mg} / \mathrm{dL}$ e $130 \mathrm{mg} / \mathrm{dL}$, respectivamente, e a mediana de $108 \mathrm{mg} / \mathrm{dL}$ (Figura $2 \mathrm{e}$ 3). Os limites mínimo e máximo de de valores entre 40 e $400 \mathrm{mg} / \mathrm{dL}$ (Figuras 2 e 3 ).

De forma geral, a venopunção pode causar estresse levando a uma elevação transitória da glicemia (WIEDMEYER et al., 2005). Esse fenômeno foi detectado no presente trabalho, sendo que nas 72 horas de acompanhamento, $100 \%$ dos animais apresentaran maiores elevações nos valores da glicose intersticial no ato da coleta do sangue venoso, para a calibração do aparelho GCMS (mediana de $74 \pm \mathrm{mg} / \mathrm{dL}$ ). Os menores valores da glicose intersticial (mediana de $40 \mathrm{mg} / \mathrm{dL}$ ) foram observados no período noturno situado entre $00 \mathrm{~h} 00 \mathrm{~min} \mathrm{e}$ 07h00min (Figuras 4 e 5).

Embora as concentrações de glicose venosa usualmente sejam maiores que as de glicose intersticial, foi observada a existência de uma boa concordância entre os valores captados no sangue com os obtidos no interstício subcutâneo (WIEDMEYER et al., 2005). Foi realizada a comparação dos valores glicêmicos com o da glicose intersticial, obtidas no mesmo momento, ou seja, o momento da calibração do GCMS e não foram observadas diferenças entre as amostras determinadas pelos dois métodos $(\mathrm{p}<0,05)$, confirmando a existência de uma boa correlação entre a glicose do interstício subcutâneo e a glicose venosa. Ainda, a título de ilustração, as medianas de todos os valores de glicose obtidos via venosa foram $82,5 \mathrm{mg} / \mathrm{dL} ; 81 \mathrm{mg} / \mathrm{dL} ; 80 \mathrm{mg} / \mathrm{dL} ; 8 \mathrm{mg} /$ $\mathrm{dL} ; 86,5 \mathrm{mg} / \mathrm{dL} ; 83 \mathrm{mg} / \mathrm{dL} ; 84 \mathrm{mg} / \mathrm{dL} ; 99,5 \mathrm{mg} / \mathrm{dl}$, para cada animal, já as que foram avaliadas pelo CGMS ${ }^{\circ}$, corresporlest ponderam respectivanis a $5 \mathrm{mg} / \mathrm{dL} ; \mathrm{e} 86 \mathrm{mg} / \mathrm{dL}$ (Figura 5). Embora esses valores tenham sido registrados no mesmo horário, todas as medianas aferidas por via venosa tiveram resultados superiores às mensurações Tealzadas pela via subcutanea, embora esta superioridade não tenha sido significante em sua quase totalidade, quando comparadas as suas médias. (Figura 5).

Ainda em análise, os animais estudados apresentaram diferchças entre si ( $\mathrm{p}<0,05$, teste de Mann-Whitney) no que tange à mediana dos valores gerais de glicose intersticial. Considerando-se que a colocação do CGMS, calibração e acompanhamento da condição de fixação dos sensores nos animais normais, apresentados no presente trabalho, foram realizads em domicílio $(n=4)$ e em ambie cor $n=4)$ e, portanto, acompanhados diuturnamente pelos 


\begin{tabular}{|c|c|c|c|c|c|c|c|c|}
\hline $\begin{array}{c}\text { VALORES REGISTRADOS } \\
\text { PELO GCMS } \Theta\end{array}$ & Cão 1 & Cão 2 & Cão 3 & Cão 4 & Cão 5 & Cão 6 & Cão 7 & Cão 8 \\
\hline Menor valor registrado & $48 \mathrm{mg} / \mathrm{dL}$ & $40 \mathrm{mg} / \mathrm{dL}$ & $40 \mathrm{mg} / \mathrm{dL}$ & $40 \mathrm{mg} / \mathrm{dL}$ & $48 \mathrm{mg} / \mathrm{dL}$ & $40 \mathrm{mg} / \mathrm{dL}$ & $40 \mathrm{mg} / \mathrm{dL}$ & $40 \mathrm{mg} / \mathrm{dL}$ \\
\hline Mediana & $78 \mathrm{mg} / \mathrm{dL}$ & $72 \mathrm{mg} / \mathrm{dL}$ & $60 \mathrm{mg} / \mathrm{dL}$ & $72 \mathrm{mg} / \mathrm{dL}$ & $82 \mathrm{mg} / \mathrm{dL}$ & $72 \mathrm{mg} / \mathrm{dL}$ & $50 \mathrm{mg} / \mathrm{dL}$ & $74 \mathrm{mg} / \mathrm{dL}$ \\
\hline Maior valor registrado & $102 \mathrm{mg} / \mathrm{dL}$ & $108 \mathrm{mg} / \mathrm{dL}$ & $94 \mathrm{mg} / \mathrm{dL}$ & $108 \mathrm{mg} / \mathrm{dL}$ & $112 \mathrm{mg} / \mathrm{dL}$ & $102 \mathrm{mg} / \mathrm{dL}$ & $114 \mathrm{mg} / \mathrm{dL}$ & $130 \mathrm{mg} / \mathrm{dL}$ \\
\hline
\end{tabular}

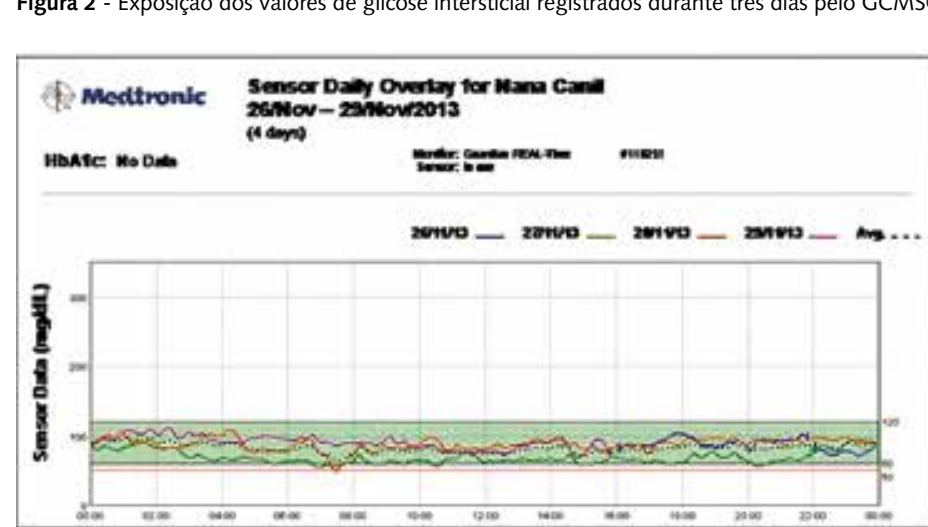

sura 3 - Curva continua da concentracăo de glicose intersticial, durante período de três

dias (72h), obtida pela utilizaçăo do CGMS๑, em cão sadio

Fonte: Casgrande K (2014)

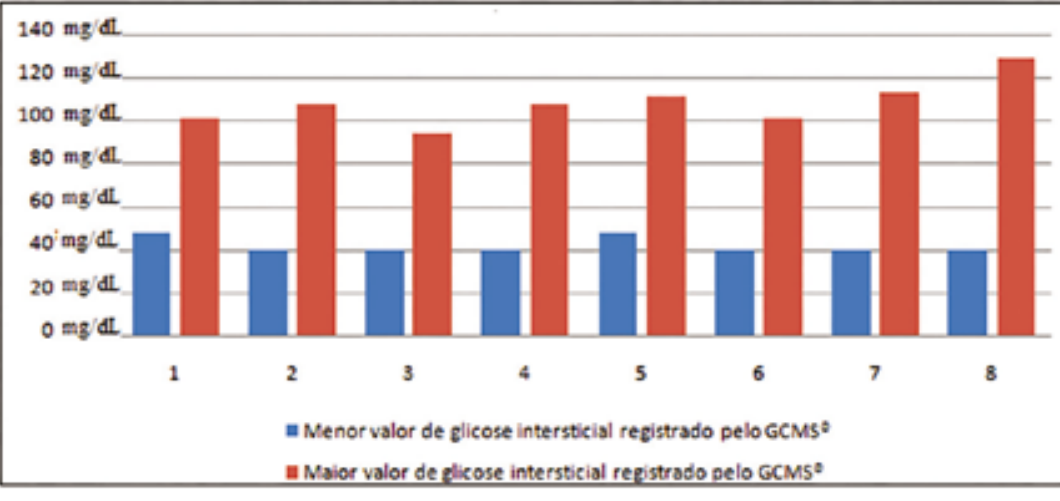

(n)

cäes sadios ( $n=8$ ) pelo sistema (GCMSe)

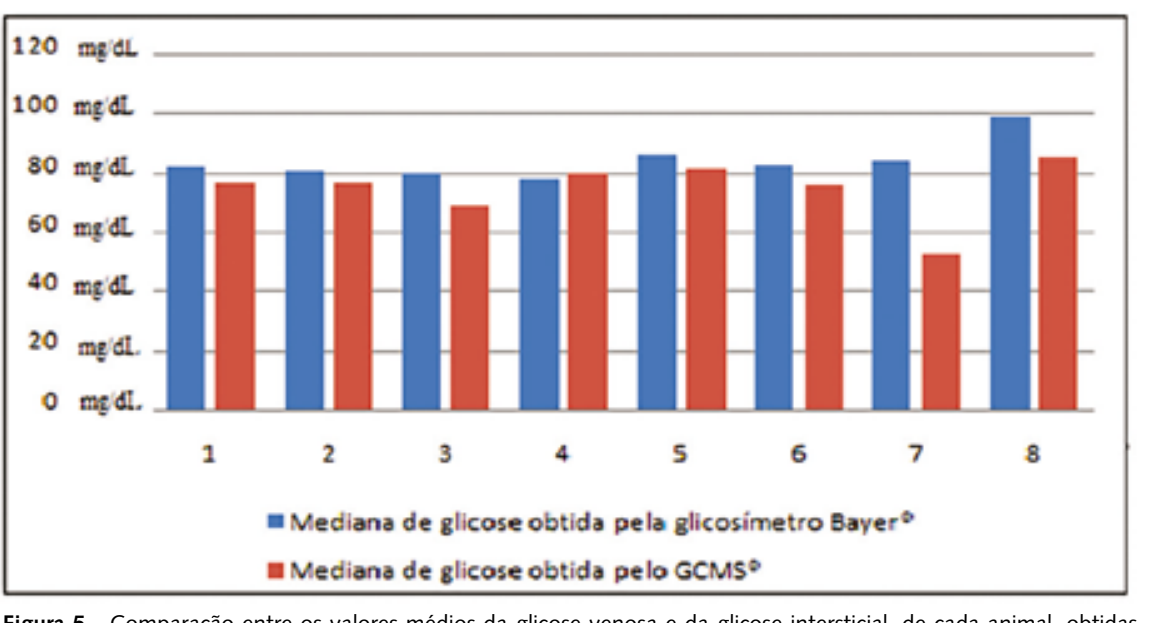

Figura 5 - Comparação entre os valores médios da glicose venosa e da gilcose intersticial, de cada animal, obtidas

simultaneamente, no momento da calibraçăo do CGMS
Fonte: Casagrande, K. (2014). responsáveis pelo projeto e pelos funcionários, e os que ficaram em ambiente doméstico, por seus tutores, foram constatados alguns percalços na obtenção de resultados e uso do aparelho, observando-se como danos maiores o fato de que dois animais desenvolveram dermatose inflamatória no local da fixação do sensor, após a retirada do mesmo, e que dois animais domiciliados tiveram os seus mesno, e que dois animas do siciltados tivera

A utilização do $\mathrm{CGMS}^{\circledR}$ foi eficaz para a avaliação da glicose intersticial de animais em âmbito doméstico, como também nos mantidos en ambiente controlado, porem ressalta-se que na avaliação domiciliar podem ocorrer alguns problemas, pois os cães estarão sob os cuidados de proprietarios leigos e não familiarizados com a tecnologia do CGMS'. Este aspecto é importante pois havera necessidade de manuseio e, principalmente, da calibração do aparelho, por meio de glicemias obtidas nas primeiras duas e seis horas após a inicialização do sensor, e a cada 12 hora até o término da monitoria da glicose intersticial. Por este manuseio faz-se necesśrin a aplicação de um treinamento prético e térico que qualifique o responśvel pelo mantseio do aparthe do seio do te bém bém que a fixaçăo do sensor do CGMS na pele do animal foi mais eficaz e duradoura quando realizada em ambiente assistido (como no canil) do que en anbiente domiciliar. Isto se deve provavelmente ao fato de que o cão em seu meio doméstico usufrui de maior mobilidade e liberdade de ações.

\section{Conclusão}

A mensuração da glicose intersticial, de forma constante é um instrumento valioso que possibilitou a análise contínu do comportamento glicêmico em cães durante as 72 horas de monitracio assim foicarcterizado um método pouco de ificar qua bes de tifcar quas de hip De como assintomáticos. Destaque-se que para o emprego do procedimento em ambiente doméstico torna-se necessário o treinamento prático e teorico do proprietário do anima pois a fixação do sensor na pele do animal mantido em ambiente doméstico foi menos eficaz quando comparada à fixação em ambiente controlado, devido a uma maior liberdade de ambulação dos animais. ๑

\section{Referências}

ABDALA, L. F. Avaliação do controle metabolico de pacientes diabéticos através do monitoramento continuo da glicose por 72 horas: estudo comparativo com métodos Faculdade de Ciências da Saúde. Brasilia, 2007.

BERG, $M$. MOONEY, C. T., P.;
Paulo: Roca, 2009 .

MURAKAMI, A. vMonCluco - sistema de monitoramento continuo de glicose 2007. (Mestrado) - Universidade de Săo Paulo. Săo Paulo, 2007.

NELSON, R. W. SALLSSURY, S. K. Neoplasias de células betas pancreáticas. In: BICHARD, de pequenos animais. São Paulo: Roca.

SEROODIO, A. T; CARVALHO, C. B.; MACHADO, J. A. Clicemia em cães (Canis familiaris) com glucômetro digital portátil e teste labor.

SURMAN, S; FLEEMAN, L. Continuous glucose monitoring in small animals. The Veternary chics or North America. Small animal practice, v. 43, n. 2, p. 381-406, 2013. WIEDMEYER, C. E.; DECLUE, A. E. Glucose monitoring in diabetic dogs and catse adapting new technology for home and hospital care. Clinics in Laboratory Medicine,

WIEDMEYER, C. E., JOHNSON, P. J., COHN, L. A., MEADOWS, R. L., KERL, M. E., for use in veterinary medicine. Diabetes Technology \& \& Therapeutics $v$. . . . . . 6. p. .885895, 2005. 